Advances in Applied Physics, Vol. 1, 2013, no. 2, 93 - 103

HIKARI Ltd, www.m-hikari.com

\title{
Spectrum of Emitted Light \\ from Sonoluminescence Bubbles
}

\author{
F. Zolfagharpour, M. H. Razbin Khalilabad, N. Satari Nikkhoo, \\ M. H. Mousavi and S. Hatampanah \\ Department of physics, University of Mohaghegh Ardabili \\ P.O. Box 179, Ardabil, Iran
}

Copyright (C) 2013 F. Zolfagharpour et al. This is an open access article distributed under the Creative Commons Attribution License, which permits unrestricted use, distribution, and reproduction in any medium, provided the original work is properly cited.

\begin{abstract}
A spherical trapped bubble at the velocity node of a resonant sound field in water, in the simplest model my be assumed to be a surface of a drop which it's excitation mode arising from small oscillation about the spherical shape. In this article by using this assumption we are trying to explain the spectrum behavior of emitted light from the bubble which trapped in a sound field in water. Also this assumption could explain dependence of the phenomena on water temperature, initial gas density of bubble and water surface tension. Also the intensity of spectrum depended on degeneracy of excited vibration state.
\end{abstract}

Subject Classification: 42.50.Fx,42.65.Re,43.35.+y, 43.35.Hl, 78.60.Mq

Keywords: Sonoluminescence, SBSL

\section{Introduction}

There are many papers [1-17] about the theory of Single Bubble Sonoluminescence but actual mechanism by which sound is converted to light remains elusive. Published papers established many of characteristics of this phenomena like occurring a sharp decrease in bubble size, emitting bluey-white light in a brief flash shorter than 100 picoseconds [10]. Despite the results that have been obtained, the exact descriptions how to produce it are rare because of the difficulty in measuring the conditions inside a pulsating bubble whose diameter 
is measured in micro-meters. It is generally agreed that the adiabatic compression of the bubble leads to very high interior temperatures, but beyond that, shocks, plasmas, ionization and photo-recombination, Bremsstrahlung radiation, and even fusion are all hotly-debated possible explanations. In this paper, we are trying to reanalysis the spectrum of emitted light from Single Bubble and dependency of light intensity on surface tension, water temperature and initial bubble density. The dependency of surface tension could explain why SL so sensitive to temperature of fluid and the light intensity increase when temperature is decrease from $40{ }^{0} \mathrm{C}$ to $0{ }^{0} \mathrm{C}$. Because of high surface tension of water we could explain why the water is friendly fluid for SL. In this view the intensity of spectrum is directly proportional with degeneracy of excited state. The mechanism of this view is in the simplest model maybe assumed the surface of bubble to be like a surface of a drop which it's excitation mode arising from small oscillation about the spherical shape. In this assumption the energy of bubble arising from the kinetic energy of gas inside the bubble and potential energy of bubble's surface but this energy assumed to be quantized and because of bubble size the exited vibration states of bubble could have very high quantized angular momentum.

\section{Theoretical formalism}

Consider a spherical bubble of radius $R_{0}$. When distorted, it's surface is convenient parameterized like liquid-drop model by the expression [18]

$$
R(\theta, \phi)=R_{\alpha}\left[1+\sum_{l=0}^{\infty} \sum_{m=-l}^{l} \alpha_{l m} Y_{l m}(\theta, \phi)\right]
$$

where $\theta$ and $\phi$ are polar angle with respect to an arbitrary apace-fixed axis and $R_{\alpha}$ is chosen to give volume of bubble. For reality, $\alpha_{l m}=(-1)^{m} \alpha_{l-m}^{*}$. Volume of bubble implies that

$$
\begin{aligned}
\frac{4 \pi}{3} R_{0}^{3}=\int_{0}^{\infty} d \phi & \int_{0}^{\pi} \sin \theta d \theta \int_{0}^{R(\theta, \phi)} r^{2} d r \\
& =\frac{4 \pi}{3} R_{\alpha}^{3}\left[1+\frac{3 \alpha_{00}}{\sqrt{4 \pi}}+\frac{2}{4 \pi} \sum_{l m} \mid \alpha_{l m}^{2}+o\left(\alpha^{2}\right)+\ldots\right]
\end{aligned}
$$

where high-order terms are neglected, assuming the deformation to be small. Deformation of the type $\alpha_{00}$ in the first order causes a volume change costing too much deformation energy and is therefore discarded in the treatment of low-lying modes. Furthermore, the potential energy of the bubble should not depend on the location of center of mass, which, to first order, is given by $\alpha_{1 m}(\mu=-1,0,1)$. By constraining the center of mass at the origin, we obtain 
$\alpha_{1 m}=0$. Hence we can write

$$
R(\theta, \phi)=\frac{R_{0}}{\xi}\left[1+\sum_{l=2}^{\infty} \sum_{m=-l}^{l} \alpha_{l m} Y_{l m}(\theta, \phi)\right],
$$

where $\xi$ is a scale factor required by the condition of bubble volume (when it emits light) and is given by

$$
\xi \approx=1+\frac{1}{4 \pi} \sum_{l=2}^{\infty} \sum_{m=-l}^{l}\left|\alpha_{l m}\right|^{2} .
$$

If we ignore the volume change to second order in the $\alpha^{\prime} s$ then $\xi$ is unitary, and we obtain

$$
R(\theta, \phi)=R_{0}\left[1+\sum_{l=2}^{\infty} \sum_{m=-l}^{l} \alpha_{l m} Y_{l m}(\theta, \phi)\right],
$$

in the quadratic approximatin, the kinetic energy is of the form

$$
T=\frac{1}{2} \sum_{l m} B_{l}\left|\alpha_{l m}\right|^{2},
$$

which

$$
B_{l}=\frac{\rho R_{0}^{5}}{l}
$$

where $\rho$ is the density and we have $\rho=\rho_{0 b} R_{0 b}^{3} / R_{0}^{3} \cdot \rho_{0 b}=1.1839 \mathrm{Kg} / \mathrm{m}^{3}$ and $R_{0 b}=5 \times 10^{-6} \mathrm{~m}$ respectively are initial bubble density and radius. This expression for the inertial parameter $B_{l}$ turns out to be quit unrealistic in the gas case and precise model is necessary for more realistic estimate. Because of distortion from sphericity the potential energy changes because the surface energy increases as a result of an increase in surface area of the bubble. this change in the potential energy from its spherical value is denoted by $\mathrm{V}$ and is given in the quadratic approximation by

$$
V=\frac{1}{2} \sum_{l m} C_{l}\left|\alpha_{l m}\right|^{2}
$$

like the liquid-drop model for the gas

$$
C_{l}=S R_{0}^{2}(l-1)(l+2) .
$$

$\mathrm{S}$ is the surface tension of bubble and for bubble in water we have

$$
S=S_{0}\left(1-\frac{T}{T_{c}}\right)^{1.2},
$$


where $\mathrm{T}$ is the water temperature in kelvin and for water $T_{c}=374{ }^{0} \mathrm{k}$ and $S_{0}=75.5 \times 10^{-3} \frac{N}{M}$. With expression 6 and 8 for $\mathrm{T}$ and $\mathrm{V}$, the Lagrangian $L=T-V$ is seen to be a sum of separate terms for each of the generalized coordinates $\alpha_{l m}$, and each of these terms is the Lagrangian of a simple harmonic oscillator. The frequency associated with the variable $\alpha_{l m}$ is

$$
\omega_{l}=\left(\frac{C_{l}}{B_{l}}\right)^{\frac{1}{2}} .
$$

So the energy eigenvalues are given by

$$
\varepsilon_{n}=\hbar \sqrt{\frac{S_{0}}{\rho_{0 b} R_{0 b}^{3}}}\left(1-\frac{T}{T_{c}}\right)^{0.6}(n(n-1)(n+2))^{\frac{1}{2}},
$$

which the degeneracy of states is $g\left(\varepsilon_{n}\right)=n(n+2)$. The density of emitting vibration mode my be proportional the density of acoustic vibration mode inside the bubble and the bubble surface acoustic vibration mode. So the density of acoustic vibration mode inside the bubble is equal to

$$
D_{\text {inside }}\left(\omega_{a}\right)=\frac{V_{b} \omega_{a}^{2}}{2 \pi^{2} v_{i n}^{3}}
$$

$V_{b}$ is the bubble volume when light started to emit from the bubble and $v_{\text {in }}$ is the speed of acoustic vibration inside the bubble. The density of bubble surface acoustic vibration mode is

$$
D_{\text {Surface }}\left(\omega_{a}\right)=\frac{2 R_{b}^{2} \omega_{a}}{\pi v_{\text {Sur }}^{2}},
$$

where $V_{b}=4 \pi R_{b}^{3} / 3$ and $v_{\text {sur }}$ is the speed of acoustic vibration on the bubble surface. The density of acoustic vibration mode could be

$$
D_{\text {acoustic }}\left(\omega_{a}\right)=D_{\text {inside }}\left(\omega_{a}\right) D_{\text {Surface }}\left(\omega_{a}\right)=\frac{4 R_{b}^{5} \omega_{a}^{3}}{3 \pi^{2} v_{\text {in }}^{3} v_{\text {Sur }}^{2}} .
$$

So the intensity of emitted light from the bubble could be proportional to

$$
D\left(\omega_{n}\right)=g\left(\omega_{n}\right) D_{\text {acoustic }}\left(\omega_{a}\right) \propto \frac{R_{b}^{5} \omega_{a}^{3}}{v_{\text {in }}^{3} v_{\text {Sur }}^{2}} n(n+2) .
$$

For wave on the surface of bubble $\omega_{a} \propto \sqrt{S}=\sqrt{S_{0}\left(1-\frac{T}{T_{c}}\right)^{1.2}}$ and $v_{i n}=\sqrt{\frac{\gamma \Re T}{M}}$. For air, we have $\gamma=1.4, M=0.02895 \mathrm{Kg} / \mathrm{mol}, \Re=8.314 \mathrm{~J} / \mathrm{Mol} . \mathrm{K}$ and for water $v_{\text {Sur }}$ parameterized as

$$
v_{\text {Sur }}=a+b T^{\prime}+c T^{2}+d T^{\prime 3}+e T^{\prime 4}+f T^{\prime 5}+g T^{\prime 6}+h T^{\prime 7}+i T^{\prime 8},
$$




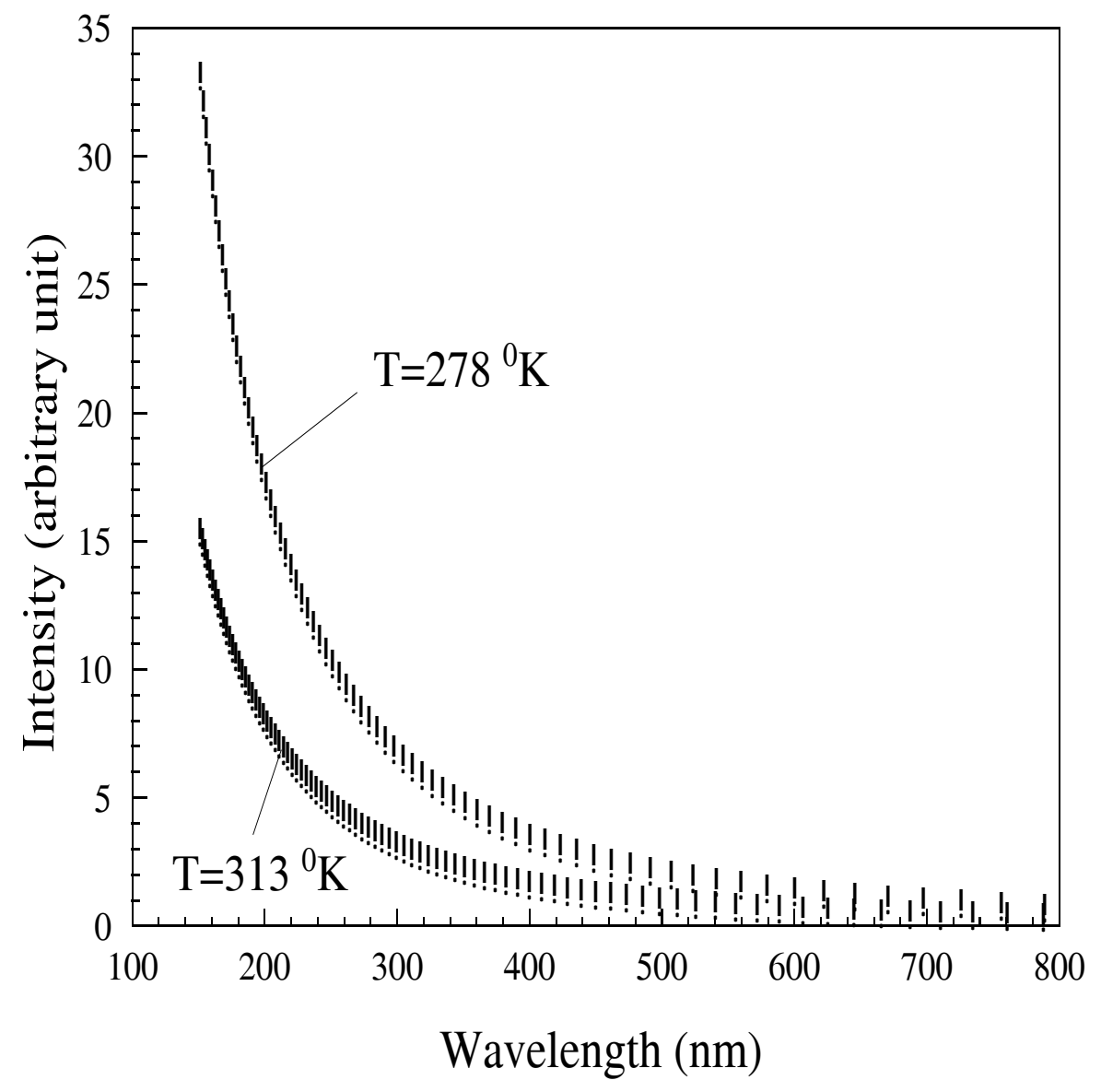

Figure 1: The extracted intensity of emitted light from bubble (arbitrary units) plotted as a function of wavelength. $\mathrm{T}$ is the water temperature.

where $T^{\prime}=T-273, a=1403.067, b=5.120365, c=-0.0871107, d=$ $0.0023998, e=-0.000088, f=0.0000021, g=-2.9677 \times 106-8, h=$ $2.06925 \times 10^{-10}$ and $i=-5.6981 \times 10^{-13}$. So we could write

$$
D\left(\omega_{n}\right) \propto \frac{R_{b}^{5}\left(M S_{0}\right)^{\frac{3}{2}}\left(1-\frac{T}{T_{c}}\right)^{1.8}}{(\gamma \Re T)^{\frac{3}{2}} v_{\text {Sur }}^{2}} n(n+2) .
$$

If we suppose that the bubble excited state is superposition of all possible angular momentum eigenvalue state and all the transition go to the grand state so in this model light intensity could be written as:

$$
I\left(\lambda_{n}\right)=C \frac{\left(M S_{0}\right)^{\frac{3}{2}}\left(1-\frac{T}{T_{c}}\right)^{1.8}}{(\gamma \Re T)^{\frac{3}{2}} v_{\text {Sur }}^{2}} n(n+2) \varepsilon_{n} .
$$

where $\lambda_{n}=h c / \varepsilon_{n}$ and $\mathrm{C}$ is a constant. 


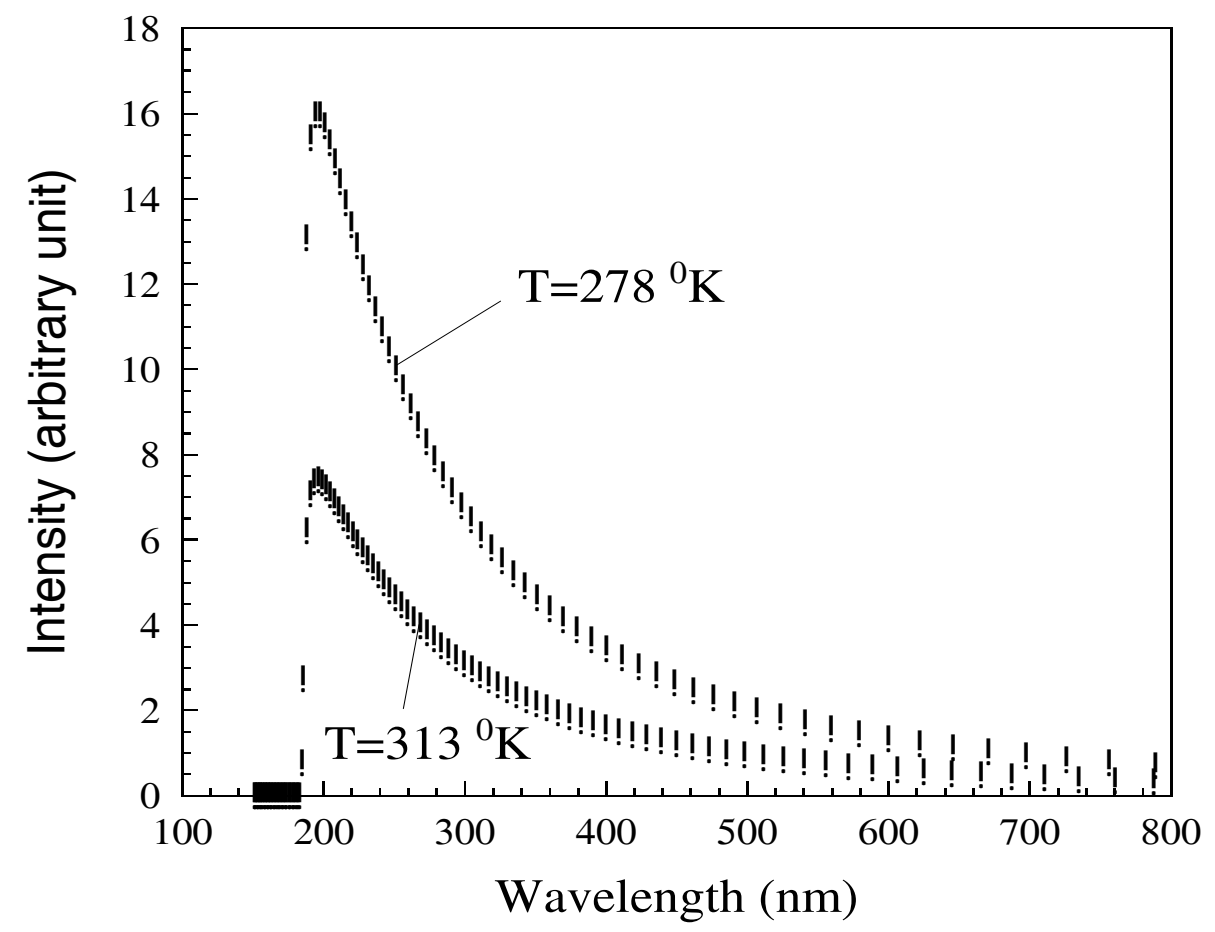

Figure 2: The extracted intensity of emitted light from bubble (arbitrary units) plotted as a function of wavelength by considering water absorbtion effect. $\mathrm{T}$ is the water temperature. 


\section{$3 \quad$ Numerical results and discussion}

We study the sonoluminescence effect in the collective bubble motion and the vibrational modes of spherical bubble, like liquid-drop model. In this model, the bubble has a collective vibrational exited state from it's spherical shape. The angular momentum of this states is quantized and very high. The maximum order of this angular momentum maybe is as high as $L^{2}=$ $l(l+1) \hbar^{2} \simeq\left(r_{0 b} m_{0 b} v_{i n}\right)^{2}$. Where $m_{0 b}$ is the mass of gas inside the bubble. Any vibration state could be sum of angular momentum eigenvalue states. During the collapsing (maybe in the end of collapsing), this state freezing to grand state. With this assumption the extracted results plotted in Figure 1 and 2. In figure 1, the intensity of emitted light from single bubble sonoluminescence (SBSL) without consideration of water absorption affect, plotted as a function of $\lambda$ according to Eq. $19(I(\lambda) / C)$ at water temperature $T=278^{0} \mathrm{~K}$ and $T=313{ }^{0} K$. In figure 2 , the measured intensity of emitted light according to

$$
I_{\text {Measured }}\left(\lambda_{n}\right)=I\left(\lambda_{n}\right) / C * e^{-\mu d}
$$

plotted. Where $\mu$ is the water Absorption coefficient which plotted in figure 3 [19]. The parameter $\mathrm{d}$ is distance that emitted light have to pass inside the water tank to com out. Here we considered $\mathrm{d}=5 \mathrm{~cm}$. Figure 3 shows that the water absorbtion coefficient is very high below the $\lambda=190 \mathrm{~nm}$, so by multiplying water absorption effect to the intensity of emitted light, the measured intensity should be different from plotted result in the figure 1. This absorbtion phenomena make a cutoff in the measured intensity which the extracted results show in the figurer 2 but the spectrum of emitted light from bubble continued below the $200 \mathrm{~nm}$. To investigating the nature of intensity below the cutoff, it needs more investigation in probability of exiting vibration modes of spherical bubble and the maximum angular momentum that the bubble could achieve. For effect of dissolved gas in the intensity of light, maybe one could say that gas dissolving gives more stability to bubble surface so during the collapsing, bubble air has less permission to dissolve in the water. So the effect of dissolved gas is like increasing the surface tension. Maybe any gas that could dissolve better, could have much role in increasing the intensity of light. In this model we considered that collapsing process is adiabatic and the maximum temperature inside the bubble in the minimum size $R_{\text {Min }} \simeq R_{0} / 5$ could reach about $2000 \mathrm{~K}$. In figure 4 the obtained result from numerical solving of one dimension heat transformation equation

$$
\frac{\partial T}{\partial t}=D \frac{\partial^{2} T}{\partial x^{2}}
$$

inside the water at distance $x=10^{-7} \mathrm{~m}$, plotted. $D=1.39 \times 10^{-7} \mathrm{~m}^{2} \mathrm{~s}^{-1}$ is the water thermal diffusivity. In initial condition at $t=0$, the temperature of 


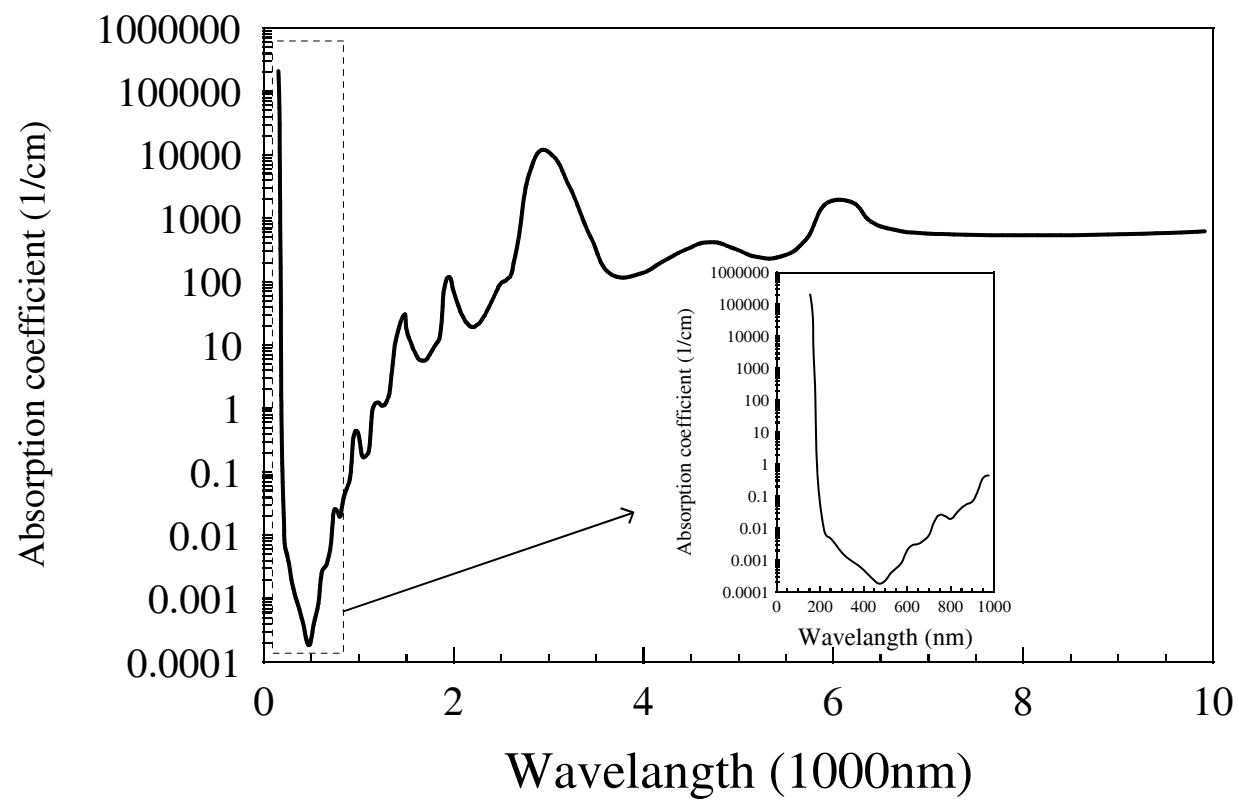

Figure 3: The visible and UV absorption coefficient of water that considered in Eq. 20. The ultraviolet, visible and infrared part of absorption coefficient plotted in the small box. Data are taken from [19].

heat reservoir and water considered respectively at $2000 \mathrm{~K}$ and $283 \mathrm{~K}$. This figure shows that rising the temperature of water surrounding the heat reservoir (bubble) at distance $x=10^{-7} \mathrm{~m}$, about one degree after flashing, needs 40 picosecond (small box in the figure 4). This result could tell us that rising of one degree of water temperature around the bubble at distance $0.1 \mu \mathrm{m}$ needs about 1 percent of total energy of bubble. So to consider this model to be reasonable, the pulse widths should be less or order of this time. We should mention to get the prices results, one should solve the three dimension of heat transfer equation for bubble and it's surrounding water. 


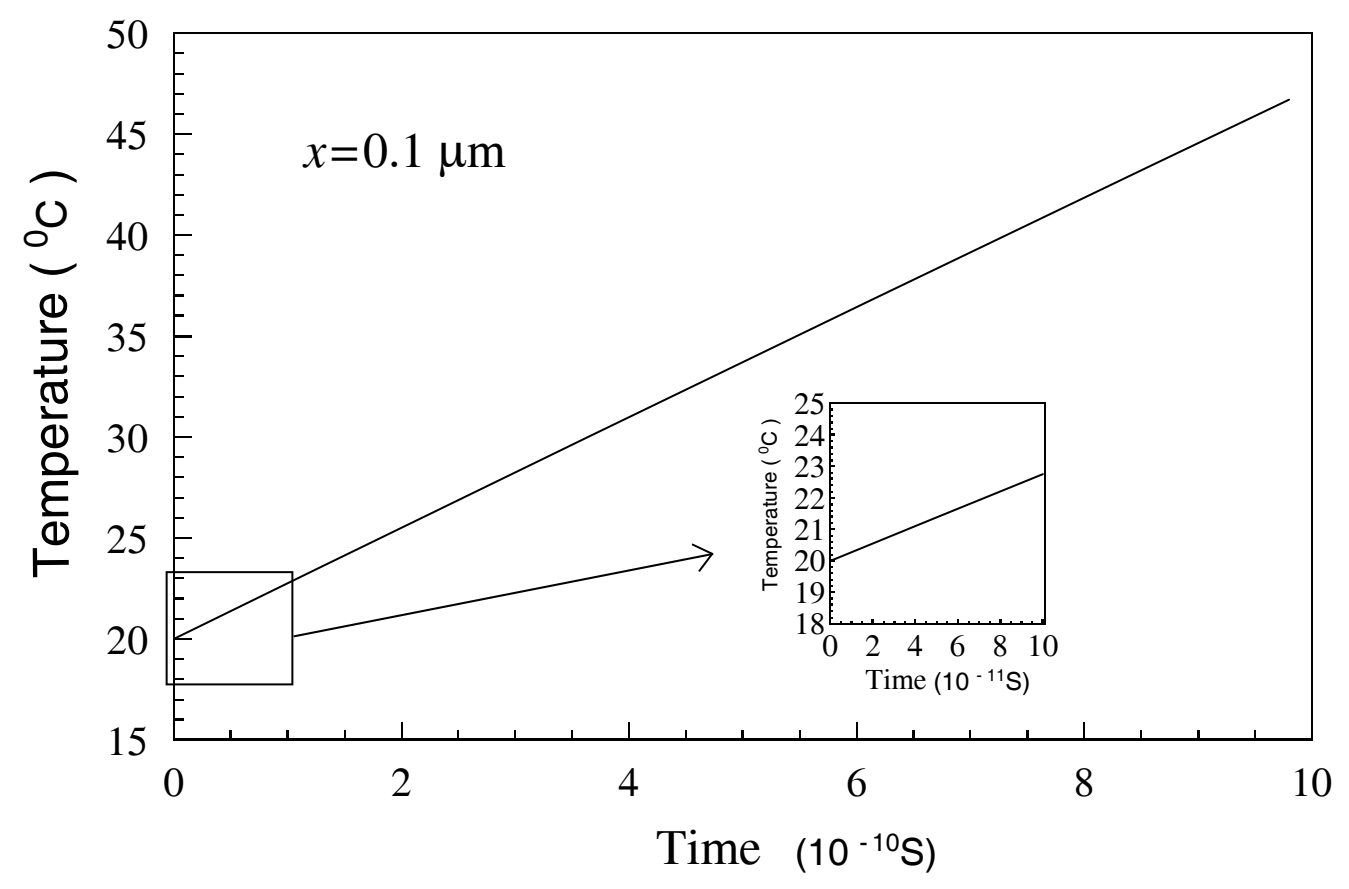

Figure 4: The temperature of water at the distance $0.1 \mu \mathrm{m}$ from the heat reservoir (bubble surface) plotted a function of time. At $t=0$ the temperature of heat reservoir (inside the bubble) and water are respectively $2000 \mathrm{~K}$ and $283 \mathrm{~K}$. The results for less than 100 picosecond showed in the small box. 


\section{References}

[1] B. P. Barber, R. A. Hiller, R. Löfstedt, S. J. Putterman, and K. R. Weninger, Phys. Rep. 281, 65 (1997).

[2] M. P. Brenner, S. Hilgenfeldt and D. Lohse, Rev. Mod. Phys. 74, 425 (2002).

[3] R. A. Hiller, S. J. Putterman, and K. R. Weninger, Phys. Rev. Lett. 80, 1090 (1998).

[4] R. Hiller, S. J. Putterman, and B. P. Barber, Phys. Rev. Lett. 69, 1182 (1992).

[5] C. Y. Ho,L. Yuan, M. C. Chu,P. T. Leung and W. Wei, Phys. Rev. E 65, 041201 (2002).

[6] D. Shapira and M. Saltmarsh, Phys. Rev. Lett. 89, 104302 (2002).

[7] G. Vazquez, C. Camara, S. J. Putterman and K. Weninger, Phys. Rev. Lett. 88, 197402 (2002).

[8] P. Mohanty and S. V. Khare, Phys. Rev. Lett. 80, 189 (1998).

[9] T. J. Matula, R. A. Roy and P. D. Mourad,W. B.McNamara and K. S. Suslick, Phys. Rev. Lett. 75, 2602 (1995).

[10] R. A. Hiller, S. J. Putterman and K. R. Weninger, Phys. Rev. Lett. 80, 1090 (1998).

[11] S. Hilgenfeldt, D. Lohse and W. C. Moss, Phys. Rev. Lett. 80, 1332 (1998).

[12] G. E. Vazquez and S. J. Putterman, Phys. Rev. Lett. 85, 3037 (2000).

[13] A. Madrazo, N. Garcia and M. Nieto-Vesperinas, Phys. Rev. Lett. 80, 4590 (1998).

[14] B. P. Barber and S. J. Putterman, Nature (London), 352, 318 (1991).

[15] R. G. Holt, D. F. Gaitan, A. A. Atchley, and J. Holzfuss, Phys. Rev. Lett. 72,1376 (1994).

[16] L. A. Crum, Phys. Today 47, 22 (1994).

[17] B. P. Barber, K. Weninger, R. Löfstedt and S. Putterman, Phys. Rev. Lett. 74, 5276 (1995).

[18] J. M. Irvine, Rep. Prog. Physics., 31, 1 (1968). 
[19] M. R. Querry, P. G. Cary, R. C. Waring, Appl. Opt., 17, 3587 (1978).

Received: February 9, 2013 